\title{
Survivability and Performance Optimization of Cellular DS-CDMA Networks Considering Recovery Decision of Base Stations
}

\author{
Kuo-Chung Chu ${ }^{*}$ and Frank Yeong-Sung Lin ${ }^{\dagger}$ \\ Department of Information Management \\ National Taiwan University, Taipei, Taiwan \\ •d5725003@im.ntu.edu.tw †yslin@im.ntu.edu.tw
}

\begin{abstract}
In this paper, we analyze the network survivability in CDMA networks considering that base stations may fail. A survivable network is modeled as mathematical optimization problem. The objective is to minimize the total blocking traffic. We apply Lagrangean relaxation as a solution approach and analyze results on call blocking rate, service rate, as well as CPU time. Experiments illustrate that the recovery of base stations is a key factor to reducing call blocking rate for the smaller base station number and for the system in the heavy loading. Time consumed for increasing the base station number is more significant than it for increasing the mobile user number.
\end{abstract}

Keywords: Base station recovery, Lagrangean relaxation, mathematical modeling, network survivability, performance evaluation, quality of service.

\section{Introduction}

Theoretically, CDMA provides no upper limit of available channels, but its capacity is bound with quality of service (Q0S) constraint. Chalmers and Solman [1] identify three impacts of mobility on QoS, including link type, movement, and mobile devices impacts. A typical infrastructure of cellular network consists of a number of components, including base stations (BS), base station controllers (BSC), mobile switching centers (MSC), home location registers (HLR) and visiting location registers (VLR), signaling system 7 (SS7), public switched telephone networks (PSTN), high-capacity trunks, etc. In the entire system, the base station which directly communicates with mobile users is a critical facility. If more base stations are deployed, there is a greater possibility of failure. A number of issues related to reliability and survivability of wireless and mobile networks are identified [2]. To analyze the impact of infrastructure component failures, a scalable approach to modeling and simulating the reliability and survivability of wireless networks is presented [3]. Even though that spare part is an alternative to tackle the problem of components failure, some natural disasters such as floods and earthquakes as well as some human factors, may crash the base station. Thus, part of the users may be out of service so that overall QoS will degrade. In terms of operation, if some base stations can be properly recovered, it enhances QoS and provides survivable service. Accordingly, base station recovery is one of the most important approaches to minimizing total system call blocking rate.

To the best of our knowledge, intensive researches have been conducted on planning of cost minimization both for general wireless survivable networks [4], and for DS-CDMA networks [5], but they focus on Iong-term planning problem. Actually, network monitoring/servicing to identify and to alleviate potential failures in state of system operation is more important. Although performance comparison of FH-CDMA and DS-CDMA for wireless survivable networks is discussed [6], relatively little work has been attempted to tackle the overall call blocking problem in conjunction with recovery decision of base station. The remainder of this paper is organized as follows. In Section 2, a mathematical problem formulation of survivable networks is proposed. Section 3 presents a solution approach to the problem based on Lagrangean relaxation. Section 4 illustrates the computational experiments. Finally, Section 5 concludes this paper.

\section{Network Survivability and Performance Modeling}

\subsection{Problem description}

A basic approach to provisioning survivability and to reducing the impact of base station failures is recovery by redundant resources or mobile base stations. An alternative is overlaying architecture by adjusting transmission power of all available/workable base stations. Figure 1 illustrates an example which consists of four base stations (BS0 BS3) and eight mobile users (M0 M7). There are two mobile users covered by each base station. In Figure 1(a), all base stations work normally (no failure) in power radius $R . \mathrm{M} 2$ and M5 is served by BS1 and BS2, respectively. Figure $1(\mathrm{~b})$ is a case of both $\mathrm{BS} 1$ and $\mathrm{BS} 2$ failures. Thus, BS0 and BS3 adjust power with $R$ ' to serving M2 and M5, respectively. However, if some of failed base stations are to be recovered, it must be a complicated decisions. Figure $1(\mathrm{c})$ and (d) is alternative to recovering one of failed base stations BS2 and BS3, 
respectively. Also, power of available base stations BS0 and BS3 is adjusted. Network survivability considering recovery decision of failed base stations, e.g. recovering $N$ of $M$ failed base statons $C(M, N)$, is a combinational optimization problem. A number of assumptions, including perfect power control, reverse link perfectly separated from the forward link, without considering fading and forward link, are given. In terms of call blocking analysis, some complicated scenarios like new, re-homing, outbound, handover calls are not dealt with.

\subsection{Performance indicators}

About the performance issues of wireless communications, Snow et al. [2] identify outage index taking into account both magnitude - the number of customers being influenced, and duration-the amount of time that service being impacted. Besides, the index focuses on two service components: registration blocking $\left(I_{R B}\right)$, when the customer is unable to initially register with the wireless network system; and call blocking $\left(I_{C B}\right)$, failures to call setup from registered customers. Based upon both $I_{R B}$ and $I_{C B}$, the following induces two performance indicators to be analyzed in this research, say service rate and call blocking rate. The detailed description of the two performance indicators is as follows,

(1) Service rate $\left(R_{S}\right)$ : No matter what causes the base stations to fail, power adjustment of available/workable base stations (integrating partial fixed base stations) is conducted. Some mobile users originally covered by the base stations are probably still out of service. This out-ofservice results in registration blocking, $R_{S}=\left(1-I_{R B}\right)$.

(2) Call blocking rate $\left(R_{B}\right)$ : The call blocking probability $\left(\mathrm{P}_{\mathrm{B}}\right)$ of mobile users is expressed by Erlang's B formula $P_{B}=B(g, c)$, where $\mathrm{g}$ is aggregate traffic, $c$ is available channel resources. Thus, call blocking rate is $R_{B}=g \cdot P_{B}=g \cdot B(g, c)$. Actually, in the survivable environment, $\mathrm{P}_{\mathrm{B}}$ can be further decomposed into two cases, a) $P_{B}=B(g, c)$, where $\mathrm{g}$ is aggregate flow of total users covered by workable base stations, and $c$ is overall available channels; b) $P_{B}=B\left(g^{\prime}, c^{\prime}\right)=1$, where $g^{\prime}$ is aggregate flow of lost traffic (out of service), and $c^{\prime}$ is zero since the system does not allocate any channels.

\subsection{Performance modeling}

The network survivability is formulated as a following mathematical optimization problem (IP) that the objective is to minimize the total call blocking rate of overall system, which is the sum of average blocking in the coverage and lost traffic $\left(g^{\prime}\right)$. Notations used to modeling the problem are listed in the Table 1. Even though the call blocking probability, $B_{j}$ of Erlang's B formula, is nondifferentiable with respect to $c_{j}$ and nonconvex with respect to $g_{j}$, but the

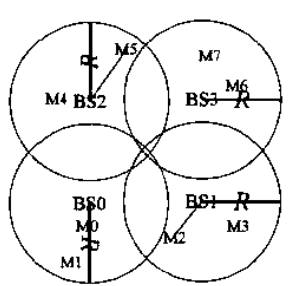

(a)

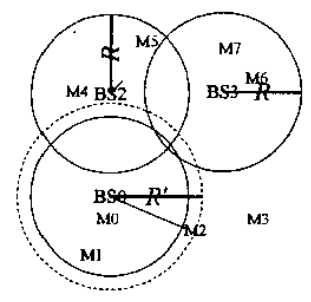

(c)

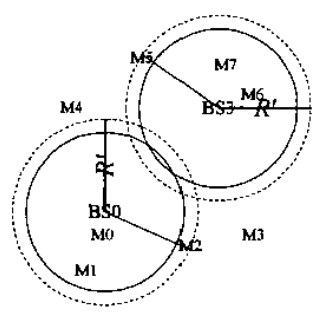

(b)

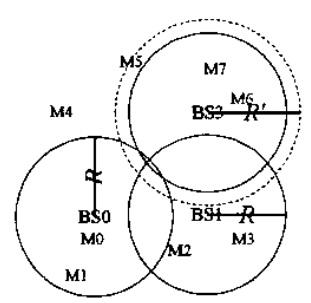

(d)
Figure 1. An example of network survivability ; (a) normal case; (b) BS1 and BS2 failures with BS0 and BS3 power adjustment ; (c) BS2 recovery with BS0 power adjustment ; (d) BS1 recovery with $\mathrm{BS} 3$ power adjustment

call blocking rate, i.e. $g_{j} \cdot B_{j}$, is a convex function of $g_{j}$ rather than $B_{j}$ alone [7].

$$
Z_{I P}=\operatorname{Min} \sum_{j \in B^{n}} g_{j} B_{j}\left(g_{j}, c_{j}\right)+g^{\prime}
$$

subject to:

$$
\begin{aligned}
& \left(\frac{E_{b}}{N_{\text {toal }}}\right)_{\text {req }} \leq \frac{\frac{S}{N_{0}}}{\left(1+\frac{1}{G} \alpha \frac{S}{N_{0}}\left(c_{j}-1\right)+\frac{1}{G} \alpha \frac{S}{N_{0}} \sum_{\substack{j \in B_{j} \\
j \neq j}}\left(\frac{\frac{r_{j}}{2}}{\operatorname{Man}\left(D_{j}, \frac{r_{f}}{2}, \sigma\right)}\right)^{T} c_{j}\right)} \\
& \sum_{\kappa \in T} \lambda_{t} z_{j t}=g_{j} \quad \forall j \in B^{\prime \prime}(1) \\
& D_{j t} z_{j t} \leq r_{j} \mu_{j t} \quad \forall j \in B^{n}, t \in T(3) \\
& \sum_{j \in B^{\prime} \cup b^{\prime}} z_{j t}=1 \quad \forall t \in T \\
& \mu_{j t} \leq f_{j} \oplus s_{j} \quad \forall j \in B, t \in T \\
& f_{j} \leq s_{j} \quad \forall j \in B(6) \\
& \sum_{j \in F} f_{j} \leq U \\
& c_{j} \leq M_{j} \quad \forall j \in B^{\prime \prime}(8) \\
& 0 \leq r_{j} \leq R_{j} \quad \forall r_{j} \in Y_{j}, j \in B^{\prime \prime}(9) \\
& B_{j}\left(g_{j}, c_{j}\right) \leq \beta_{j} \quad \forall j \in B^{\prime \prime}(10) \\
& z_{j t}=0 \text { or } 1 \quad \forall j \in B^{\prime \prime}, t \in T(11) \\
& f_{j}=0 \text { or } 1 \quad \forall j \in B(12) \\
& c_{j} \in Z^{+} \forall j \in B^{\prime \prime}
\end{aligned}
$$


Constraint (1) ensures that each traffic demand is served by a base station in the required QoS. Constraint (2) checks aggregate flow of each base station $j \in B^{\prime \prime}$. Constraint (3) requires that a mobile user must be in the coverage of a base station before it is to be served by that base station. Constraint (4) is to guarantee that each mobile user can be homed to no more than one base station. Constraint (5) ensures that $\mu_{j t}=1$ only if $f_{j} \oplus s_{j}=1$ in each of two cases, a) base station $j$ is failed $\left(s_{j}=1\right)$ and to be fixed $\left(f_{j}=1\right)$; b) base station $j$ is $\operatorname{good}\left(s_{j}=0\right)$ and no fix is needed $\left(f_{f}=0\right)$. Constraint (6) ensures that the decision variable of fixing base stations is set to 1 only if $s_{f}=1$. Constraint (7) guarantees that total number of fixed base stations is less than predefined threshold $U$. Constraint (8) is to ensure that the number of users who can be active at the same time in a base station is no greater than upper bound $M_{j}$. Constraint (9) is to ensure that transmission power radius of each base station $j \in B$ " is between 0 and $R_{j}$. Constraint (10) requires that a base station serves its slave mobile users under predefined threshold of call blocking probability. Constraints (11) and (12) are to enforce the binary property of the decision variables. Finally, constraint (13) denotes the integer property of decision variables for channel allocation.

\section{Solution Approach}

\subsection{Lagrangean relaxation}

The approach to solving the problem (IP) is Lagrangean relaxation [8] [9], which includes the procedures that relax complicating constraints, multiply the relaxed constraints by corresponding Lagrangean multipliers, and add them to the primal objective function. Based on above procedures, we transform the primal optimization problem (IP) into the following Lagrangean relaxation problem (LR) where Constraints (1), (2), (3), (5), (6) are relaxed. Furthermore, LR can be decomposed into three independent subproblems: 1) channel assignment, power control subproblem and capacity management subproblem; 2) admission control subproblem; 3) base station recovery subproblem.

$$
\begin{aligned}
& Z_{D}\left(v_{j}^{1}, v_{j}^{2}, v_{j t}^{3}, v_{j t}^{4}, v_{j}^{5}\right)=\min \sum_{j \in B^{n}} g_{j} B_{j}\left(g_{j}, \hat{c}_{j}\right)+\sum_{r \in T} \lambda_{t}\left(1-\sum_{j \in B^{t}} z_{j t}\right) \\
& \sum_{j \in B^{n}} v_{j}^{1}\left(\left(\frac{E_{b}}{N_{\text {total }}}\right)_{\text {req }}+\left(\frac{E_{b}}{N_{\text {total }}}\right)_{\text {req }} \frac{1}{G} \alpha \frac{S}{N_{0}}\right. \\
& \left.\left(\left(a_{j}-1\right)+\sum_{\substack{j^{\prime} \in B^{*} \\
j^{*} \neq j}}\left(\frac{\frac{r_{f}}{2}}{\operatorname{Mar}\left(D_{i t^{\prime}}-\frac{r_{i}}{2}, \infty\right)}\right)^{z} c_{j^{+}}\right)-\frac{S}{N_{0}}\right) \\
& +\sum_{j \in B^{n}} v_{j}^{2}\left(\sum_{r \in T} \lambda_{\mathrm{r}} z_{j t}-g_{j}\right)+\sum_{r \in T} \sum_{j \in B^{*}} v_{j t}^{3}\left(D_{j t} z_{j t}-r_{j} \mu_{j t}\right) \\
& +\sum_{t \in T} \sum_{j \in B} v_{j t}^{4}\left(\mu_{j t}-\left(1-f_{j}\right)\left(1-s_{j}\right)-f_{j} \cdot s_{j}\right)+\sum_{j \in B} v_{j}^{5}\left(f_{j}-s_{j}\right)
\end{aligned}
$$

\begin{tabular}{|c|c|}
\hline Notation & Description \\
\hline$B$ & The set of base stations \\
\hline$F$ & The set of failed base stations, $F \subset B$ \\
\hline$U$ & Predefined threshold of total fixed base stations \\
\hline$F^{\prime}$ & The set of fixed base stations, $F^{\prime} \subset F,|F|=U$ \\
\hline$B^{\prime}$ & The set of available base stations, $B=B-F$ \\
\hline$B^{\prime \prime}$ & The set of workable base stations, $B "=B \cup F^{\circ}$ \\
\hline$b^{\prime}$ & $\begin{array}{l}\text { The artificial base station to carry the rejected call when } \\
\text { decides to reject the call }\end{array}$ \\
\hline$T$ & The set of mobile users \\
\hline$B_{j}$ & Call blocking probability in base station $j, j \in B$ \\
\hline$M_{j}$ & $\begin{array}{l}\text { Upper bound on the number of users that can active at the } \\
\text { same time at base station } j, j \in B\end{array}$ \\
\hline$R_{j}$ & $\begin{array}{l}\text { Upper bound on the transmission power radius of base } \\
\text { station } j, j \in B\end{array}$ \\
\hline$\beta_{j}$ & $\begin{array}{l}\text { Threshold of call blocking probability for each base station } \\
j, j \in B\end{array}$ \\
\hline$c_{j}$ & $\begin{array}{l}\text { The number of users who can be active at the same time in } \\
\text { the base station } j, j \in B\end{array}$ \\
\hline$r_{j}$ & Transmission power radius of base station $j, j \in B$ \\
\hline$Y_{j}$ & The set of transmission radius of base station $j$ \\
\hline$g_{j}$ & Aggregate flow at base station $j, j \in B^{\prime \prime}$ \\
\hline$g^{\prime}$ & $\begin{array}{l}\text { Aggregate flow of mobile users not served by } B \text { ", where } \\
g=\sum_{1 \in \mathrm{T}} \lambda_{t} \sum_{\mathrm{j} \in \mathrm{B}}\left(1-z_{j j}\right)\end{array}$ \\
\hline$S$ & $\begin{array}{l}\text { The power that a base station received from a mobile user } \\
\text { that is homed to the base station with perfect power control }\end{array}$ \\
\hline$E_{b}$ & The energy that BS received \\
\hline$N_{\text {Total }}$ & Total noise \\
\hline ఐ & A small number \\
\hline$G$ & The processing gain \\
\hline$N_{0}$ & The background noise \\
\hline$\alpha$ & Voice activity factor \\
\hline$\tau$ & Attenuation factor \\
\hline$\lambda_{1}$ & The traffic requirement of mobile station $t$ (in Erlang), $t \in T$ \\
\hline$D_{j,}$ & Distance between base station $j$ and mobile user $t$ \\
\hline$D_{j i}$ & Distance between base station $j$ and $j^{\prime}$ \\
\hline$\mu_{j}$ & $\begin{array}{l}\text { Coverage indicator function which is } 1 \text { if mobile user } t \text { can } \\
\text { be served by base station } j \text { and } 0 \text { otherwise, } j \in B\end{array}$ \\
\hline$z_{j r}$ & $\begin{array}{l}\text { Granting decision variable which is } 1 \text { if mobile user } t \text { is } \\
\text { serviced by base station } j \text { and } 0 \text { otherwise, } j \in B\end{array}$ \\
\hline$s_{j}$ & $\begin{array}{l}\text { Intial indicator function of base station } j \in B \text {, which is } 1 \text { if } \\
\text { base station is failure and } 0 \text { otherwise }\end{array}$ \\
\hline$f_{j}$ & $\begin{array}{l}\text { Decision variable of fixing failed base station } j \in B \text { or not, } \\
\text { which is } 1 \text { if broken base station is fixed and } 0 \text { otherwise }\end{array}$ \\
\hline$f_{j} \oplus s_{j}$ & Exclusive-or of $f_{j}$ and $s_{j}$ expressed by $(1-f)\left(1-s_{j}\right)$ \\
\hline
\end{tabular}

subject to: (4), (7)-(13).
Table 1. Description of notation

(1) channel assignment, power control, and capacity management subproblem: related to $c_{j}, r_{j}$, and $g_{j}$

$$
\begin{aligned}
& Z_{S U B 1}=\min \sum_{j \in B^{*}}\left(g_{j} B_{j}\left(g_{j}, \hat{c}_{j}\right)+\left(\frac{E_{b}}{N_{\text {total }}}\right)_{\text {req }} \frac{1}{G} \alpha \frac{S}{N_{0}} v_{j}^{\prime}\right. \\
& \left(\hat{c}_{j}+\sum_{\substack{j \in B^{*} \\
j \neq j}}\left(\frac{\frac{r}{2}}{\operatorname{Mar}\left(D, D_{j t}-\frac{r}{2}, \sigma\right)}\right)^{x} \hat{c}_{j}\right)+\sum_{\in \in T}\left(v_{j t}^{4}-v_{j r}^{3} r_{j}\right) \mu_{j t}
\end{aligned}
$$


$\left.-v_{j}^{2} g_{j}+v_{j}^{1}\left(\left(\frac{E_{b}}{N_{\text {totat }}}\right)_{\text {req }}-\frac{S}{N_{0}}-\left(\frac{E_{b}}{N_{\text {total }}}\right)_{\text {req }} \frac{1}{G} \alpha \frac{S}{N_{0}}\right)\right)$

subject to: (8) (9) (10) (13).

Problem (SUB1) can be decomposed into $\left|B^{\prime}\right|$ subproblems since the value of $r_{j}$ and $c_{j}$ is discrete and limited. To get an optimal solution, we just exhaustively search for all possible combinations of $c_{j}, r_{j}$ and $g_{j}$.

(2) admission control subproblem: related to $z_{\mu t}$

$Z_{S U B 2}=\min \sum_{t \in T} \lambda_{i}\left(1-\sum_{j \in B^{n}} z_{j t}\right)+\sum_{j \in B^{n}} v_{j}^{2} \sum_{t \in T} \lambda_{7} z_{j t}+\sum_{t \in T} \sum_{j \in B^{n}} v_{j t}^{3} D_{j t} z_{j t}$

$=\min \sum_{i \in T} \sum_{j \in B^{n}}\left(v_{j t}^{3} D_{j t}+\left(v_{j}^{2}-1\right) \lambda_{f}\right) z_{j t}+\sum_{t \in T} \lambda_{t}$

subject to: (4) and (11).

In (SUB2), the second term $\sum_{\mathrm{t} \in \mathrm{T}} \lambda_{\mathrm{r}}=|\mathrm{T}| \times \lambda_{t}$ is a constant, total aggregate traffic in the system, which can be dropped and added back to the optimal value. Then (SUB2) can be decomposed into $\left|B^{\prime \prime}\right| \times|T|$ independent subproblems for each $l_{j t}=\left(v_{j t}^{2} D_{j t}+\left(v_{j}^{3}-1\right) \lambda_{t}\right)$ where $j \in B$ " and $t \in T$. To get the minimal value of problem (SUB2), we assign either $z_{j t}=1$ for the minimal value of $l_{j t}$, or $z_{j t}=0$ otherwise.

(3) base station recovery subproblem: related to $f_{j}$

$$
\begin{aligned}
& Z_{S U B 3}=\min \sum_{i \in T} \sum_{j \in B} v_{j t}^{4}\left(-\left(1-f_{j}\right)\left(1-s_{j}\right)-f_{j} \cdot s_{j}\right) \\
& +\sum_{j \in B^{n}} v_{j}^{5}\left(f_{j}-s_{j}\right)=\sum_{j \in B}\left(\sum_{i \in T} v_{j t}^{4}\left(1-2 s_{j}\right)+v_{j}^{5}\right) f_{j} \\
& +\sum_{j \in B}\left(\left(\sum_{i \in T} v_{j t}^{4}-v_{j}^{5}\right) s_{j}-\sum_{i \in T} v_{j t}^{4}\right)
\end{aligned}
$$

subject to: (7), (12).

$$
\text { In (SUB3), the second term } \sum_{j \in B}\left(\left(\sum_{r \in T} v_{j t}^{4}-v_{j}^{5}\right) s_{j}-\sum_{\kappa \in T} v_{j t}^{4}\right)
$$

is a constant that can be calculated easily. Then, let $k_{j}=\sum_{i \in \mathcal{T}} v_{j l}^{4}\left(1-2 s_{j}\right)+v_{j}^{5}$ so. that (SUB3) can be decomposed into $|B|$ independent subproblems for each $k_{j}$. To get a minimal value of problem (SUB3), we assign either $f_{j}=1$ for first $\mid U$ minimal value of $k_{j}$ (for constraint (7)) at most, or $f_{j}=0$ otherwise.

\subsection{Lagrangean dual problem and subgradient method}

According to the weak Lagrangean duality theorem [10], for any $\left(v_{j}^{1}, v_{j t}^{3}, v_{j t}^{4}, v_{j}^{5}\right) \geq 0$ and $v_{j}^{2}$, the objective value of $Z_{D}\left(v_{j}^{1}, v_{j}^{2}, v_{j t}^{3}, v_{j r}^{4}, v_{j}^{5}\right)$ is a lower bound on $Z_{I P}$. Based on Problem (LR), the following dual problem (D) is constructed to calculate the tightest lower bound.
Table 2. Given parameters

\begin{tabular}{cc}
\hline \hline Notation & Value \\
\hline$S / N_{0}$ & $7 \mathrm{db}$ \\
$E b / N_{\text {total }}$ & $6 \mathrm{db}$ \\
$M_{j}$ & 120 \\
$\tau$ & 4 \\
$G$ & 156.25 \\
$a_{t}$ & 10 \\
$\alpha$ & 0.75 \\
\hline \hline
\end{tabular}

$$
Z_{p}=\max Z_{D}\left(v_{j}^{1}, v_{j}^{2}, v_{j}^{3}, v_{j s}^{4}, v_{j}^{5}\right)
$$

subject to: $\left(v_{i}^{1}, v_{j l}^{3}, v_{j l}^{4}, v_{j}^{5}\right) \geq 0$ and $v_{j}^{2}$.

Then, a subgradient method is applied to solving the dual problem. Let the vector $S$ be a subgradient of $Z_{D}\left(v_{j}^{1}, v_{j}^{2}, v_{j t}^{3}, v_{j t}^{4}, v_{j}^{5}\right)$ at $\left(v_{j}^{1}, v_{j}^{2}, v_{j t}^{3}, v_{j t}^{4}, v_{j}^{5}\right)$. In iteration $\mathrm{k}$ of subgradient optimization procedure, the multiplier vector $\pi$ is updated by $\pi^{k+1}=\pi^{k}+t^{k} S^{k}$, in which $t^{k}$ is a step size determined by $t^{k}=\delta\left(Z_{I P}^{*}-Z_{D}\left(\pi^{k}\right)\right) /\left\|S^{k}\right\|^{2}$, where $Z_{I P}^{*}$ is an upper bound on the primal objective function value after iteration $\mathrm{k}$, and $\delta$ is a constant where $0 \leq \delta \leq 2$.

\subsection{Getting primal feasible solutions}

This research applies both Lagrangean relaxation and subgradient method to solving the problem of network survivability and performance modeling. The procedure is to get a better primal feasible solution in the process of iteratively solving dual problem. We develop an algorithm A to getting primal feasible solutions, it needs to be checked if solutions satisfy all constraints relaxed before.

\section{[Algorithm A]}

Step 1.Check QoS constraint (1) for each base station $j$. Adjust the transmission power radius $r_{j}$ down if the QoS constraint is still violated, or go to Step 2 otherwise.

Step 2. Compute the aggregate traffic flow $g_{j}$ of each base station $\mathrm{j}$ based on the transmission power radius $r$ determined in Step 1.

Step 3.Check the call blocking constraint $B_{j}\left(g_{j}, c_{j}\right) \leq \beta_{j}$. Assign the available channel $c_{j}$ up to meet the call blocking requirements $\beta_{\dot{p}}$ if the call blocking constraint is still violated, or go to Step 4 otherwise.

Step 4. Adjust the transmission power radius $r_{j}$ to the extent for just far enough to cover all mobile users in each base station $j$.

Step 5. Calculate the whole blocking traffic in the system that is based upon the decision variables, including $c_{j}, r_{j}, g_{j}$, and $z_{j t}$, solved in previous steps.

Step 6. End algorithm. 


\section{Computational Experiments}

\subsection{Experimental environment}

Parameters are given in Table 2. Algorithm implementation and platform running on is coded in $\mathrm{C}$ and is PC with INTEL ${ }^{\mathrm{TM}} \mathrm{P} 4-1.6 \mathrm{GHZ}$ CPU, respectively. We evaluate the algorithm for 5 cases of base station number (BS \#) $-40,80,120,160,200$, and 6 cases of mobile user number (MU \#) - 500,1000, 1500, 2000, 2500, 3000. Locations of BS as well as MU are generated in uniform distribution. The number of failed base stations $(\mid F)$ is given to one-tenth of total base stations. BS recovery ratios (BSRR) are given to $0.00,0.25,0.50,0.75$. The limit of iterations for Lagrangean relaxation approach is 1000 , and the improvement counter is 25 . The parameter $\delta$ adopted in the subgradient method is initialized to be 2 and halved when the dual objective function value does not improve for 25 iterations. Besides, predefined threshold of call blocking probability for each base station is given with $\beta_{j}=0.03$.

\subsection{Performance analysis}

The analysis focuses on $\mathrm{MU} \#=3000$, depicted in Figure 2, and BS \# $=200$, depicted in Figure 3, comparing with a number of BS and MU, respectively. Experiments calculate with all near-optimal solutions in gap less than $0.001 \%$. Experimental results include call blocking rate (upper bound of $Z_{\mathbb{P}}$ ), service rate, and CPU time.

(1) Call blocking rate $\left(R_{B}\right)$ : In case of $M U \# 3000, R_{B}$ is insignificant difference among a number of BS, Figure 2(a). In case of BS \# 200, Figure 3(a), it is a montonically increasing function of $\mathrm{MU} \#$. The number of $\mathrm{MU}$ significantly affects $R_{B}$. To analyze the impact of BSRR on $R_{B}$, for each of increasing $R_{B}$ calculated is compared with normal case (no BS failure). In Figure 2(b), the case of $\mathrm{MU} \# 3000$, increasing $R_{B}$ is varied from $15 \%$ to $71 \%$ in case of BS \# 40, while it is in the range from $10 \%$ to $44 \%$ in case of $B S \# 200$. BSRR is a key factor to reducing $R_{B}$ for small number of $B S$. In Figure 3(b), the case of BS $\# 200$, the maximum of increasing $R_{B}$ is up to $27 \%$ in case of $\mathrm{MU} \# 500$, while the increasing is varied from $9 \%$ to $44 \%$ in case of both MU \# 1500 and MU \# 3000 . BSRR is the more important factor to reducing $R_{B}$ when the system is in the heavy loading.

(2) Service rate( $\left.R_{S}\right)$ : Obviously, $R_{S}$ is a montonically increasing function of BSRR. However, it is insignificant impacts of BSRR on $R_{S}$ in all cases of BS \# and MU \# in Figure 2(c) and Figure 3(c), respectively. No matter which case is calculated, $R_{S}$ is in the range from 0.92 to 0.98 .

(3) CPU time: Time consumed is to run Lagrangean relaxation procedure up to 1000 iterations. All experiments are converged in less than 1000 iterations. Since the survivability analysis focues on avaible base stations, the more BSRR is required, the more CPU is consumed. Also, it is a increasing function of $\mathrm{MU}$ \# and BS \#. In case of MU \# 3000, Figure 2(d), CPU consumed is from 8 to 16 seconds in the smallest BS \# 40, while it calculates with 209 to 556 seconds in the largest BS \# 200. In case of BS \# 200, Figure 3(d), CPU consumed is from 57 to 117 seconds in the smallest MU \# 500, while it calculates with 209 to 556 seconds in the largest MU \# 3000. Time consumed is more significant when the number of $\mathrm{BS}$ is increasing than MU is increasing.

\section{Conclusions}

This paper investigates the survivability problem of DS-CDMA networks. Network survivability network is modeled as performance optimization problem, failures and recovery decisions of base stations are considered as well. Proposed model in conjunction with developed algorithm recovers some of the failed base stations to guarantee sustainable services as well as to assure quality of service. Experiments illustrate that the recovery of base stations is a key factor to reducing call blocking rate for the smaller number of BS as well as the system in the heavy loading. Time consumed is more significant when the number of BS is increasing than $\mathrm{MU}$ is increasing. Based on the proposed approach, we believe that a more flexible and comprehensive model can further be considered to decide where mobile/portable base stations should be allocated in dynamic user requirements.

\section{References}

[1] D. Chalmers and M. Solman, "A survey of quality of service in mobile computing environments," IEEE Communications Surveys, 2-Quarter, 1999, pp. 2-10.

[2] A.P. Snow, U. Varshney, and A.D. Malloy, "Reliability and survivability of wireless and mobile networks," IEEE Computer, vol. 33, Issue: 7, pp. 49-55, July 2000.

[3] N. Dimitriou, G. Sfikas, and R. Tafazolli, "Call admission policies for UMTS," in Proc. IEEE VTC, vol. 2, pp. 1420$1424,2000$.

[4] F. Y.-S. Lin and C.-Y. Lin, "Integrated planning and management of survivable wireless communications networks," in Proc. APCC/OECC, vol. 1, pp. $541-544$, 1999.

[5] Kuo-Chung Chu, Frank Yeong-Sung Lin, and Shih-Hao Lee, "Intergrated planning and capacity management of survivable DS-CDMA networks", accepted for publication, IEEE ICNSC, Taipei, Taiwan, March 21-23, 2004.

[6] S. Chuprun, C.S. Bergstrom, "Comparison of FH/CDMA and DS/CDMA for wireless survivable networks," in Proc. IEEE GLOBECOM, vol. 3, pp.1823 -1827, 1998.

[7] K. R. Krishnan, "The convexity of loss rate in an Erlang loss system and sojourn in an Erlang delay system with respect to arrival and service rates," IEEE Trans on Communications, 38-9, pp. 1314-1316, Sep. 1990.

[8] A. M. Geoffrion, "Lagrangean relaxation and its use in integer programming", Math. Programming Study, vol. 2, pp. 82-114, 1974.

[9] M. L. Fisher, "The Lagrangian relaxation method for solving integer programming problems", Management Science, vol. 27, pp.1-18, 1981.

[10] H. Held, P. Wolfe and H. D. Crowder, "Validation of subgradient optimization", Math. Programming, vol. 6, pp. 62-88, 1974. 


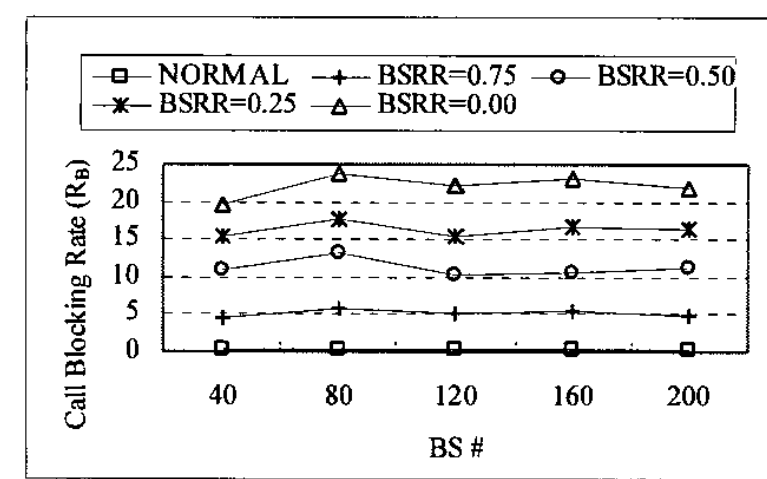

(a) Call blocking rate with respective to BSRR.

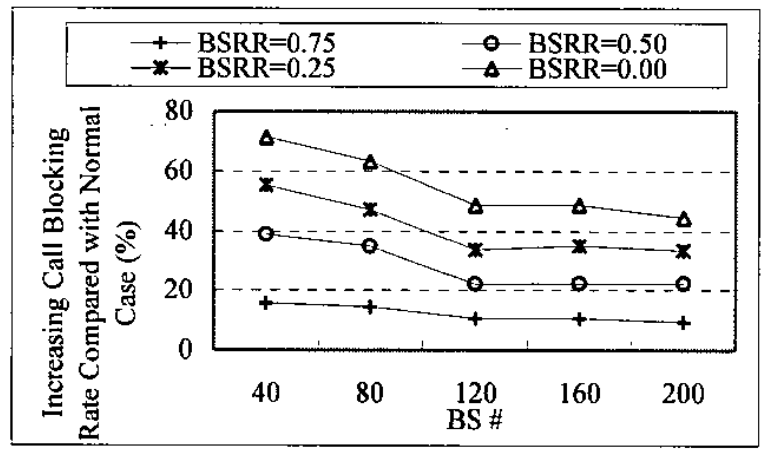

(b) Increasing call blocking rate compared with normal case.

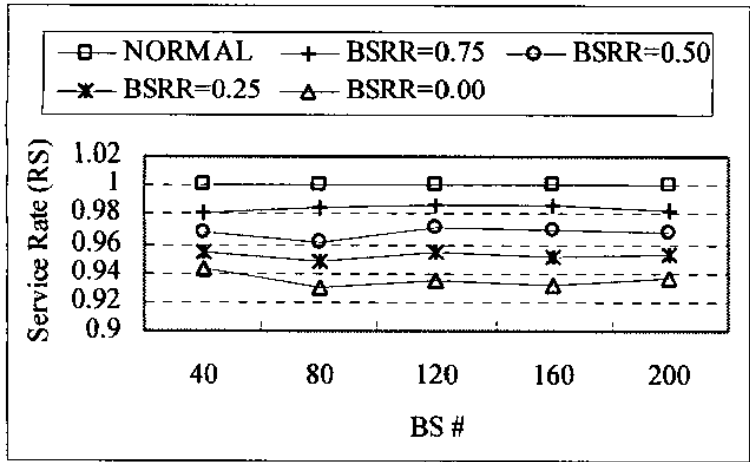

(c) Service rate with respective to BSRR.

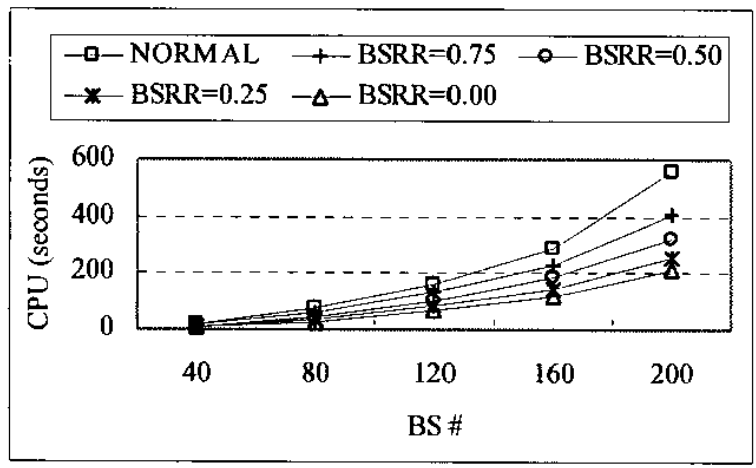

(d) CPU time consumed with respective to BSRR.

Figure 2. Experiment results of $M U=3000$ compared with a number of BS

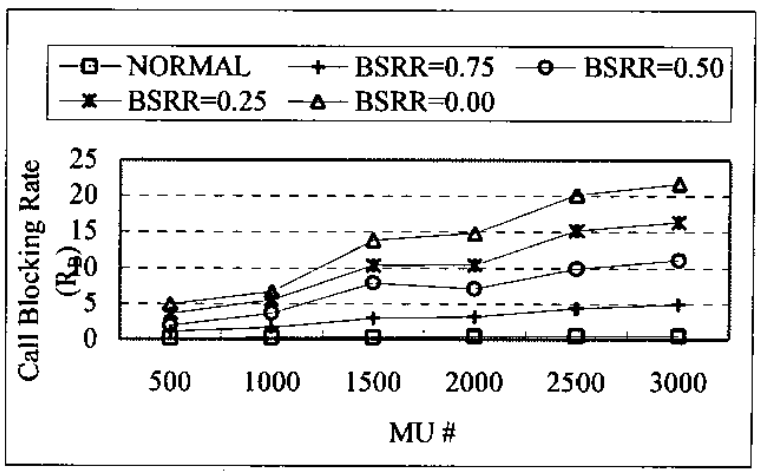

(a) Call blocking rate with respective to BSRR.

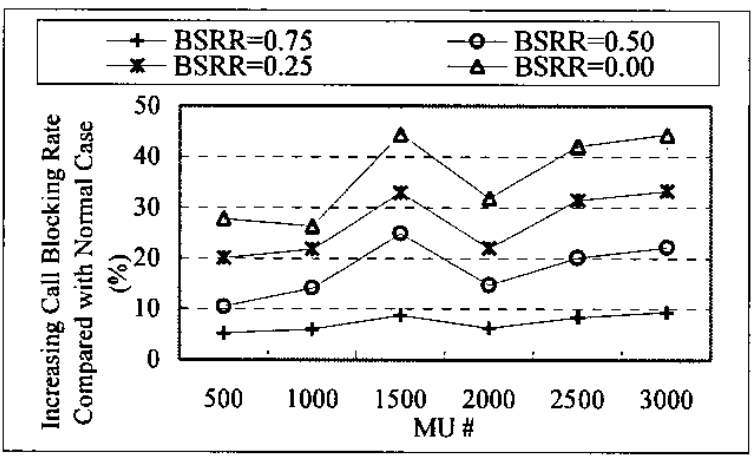

(b) Increasing call blocking rate compared with normal case.

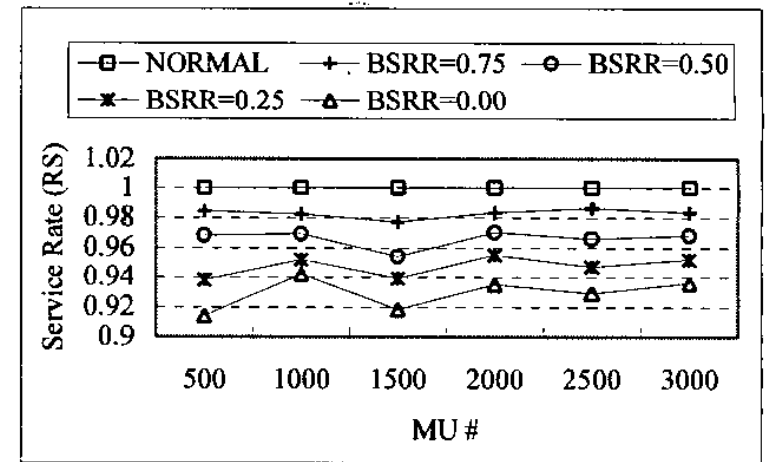

(c) Service rate with respective to BSRR.

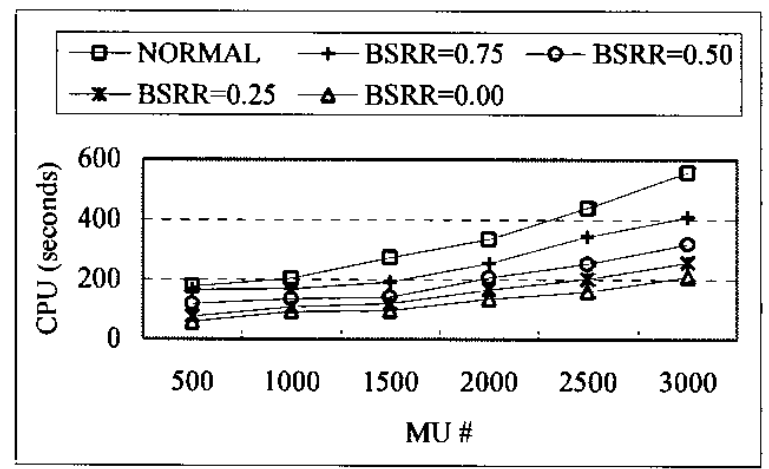

(d) CPU time consumed with respective to BSRR.

Figure 3. Experiment results of $B S=200$ compared with a number of $\mathrm{MU}$ 